P. S. GRANT, D.P. FURKERT* M. A. BRIMBLE* (UNIVERSITY OF AUCKLAND AND MAURICE WILKINS CENTRE FOR MOLECULAR BIODISCOVERY, AUCKLAND, NEW ZEALAN D )

Total Synthesis of $( \pm)$-Leonuketal

Org. Lett. 2020, 22, 8735-8740, DOI: 10.1021/acs.orglett.0c03364.

\title{
Synthesis of $( \pm)$-Leonuketal
}

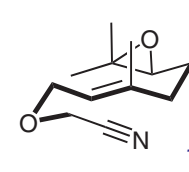

A

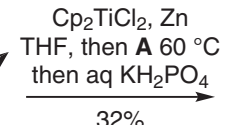

Ti-mediated cyclization cascade

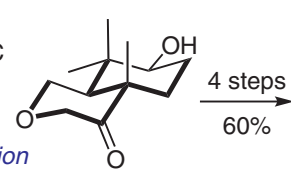

B

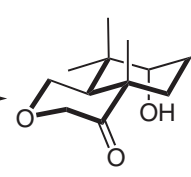

C
1. $\mathrm{TsNHNH}_{2}$

PPTS (5 mol\%), THF

2. MeLi, $\mathrm{Et}_{2} \mathrm{O}-\mathrm{THF}-\mathrm{DME}$ $(2: 2: 1), 0{ }^{\circ} \mathrm{C}$

Shapiro reaction-fragmentation

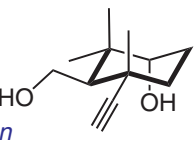

D

1. TIPSOTf, DIPEA $\mathrm{CH}_{2} \mathrm{Cl}_{2}, 0^{\circ} \mathrm{C}$

2. $\mathrm{MeLi}, \mathrm{Et}_{2} \mathrm{O}-\mathrm{DME}$

(2.2:1), -78 to $0^{\circ} \mathrm{C}$

then $\left(\mathrm{CH}_{2} \mathrm{O}\right)_{n},-78^{\circ} \mathrm{C}$ to $\mathrm{rt}$.

3. $\mathrm{Et}_{3} \mathrm{~N}, \mathrm{MsCl}, \mathrm{CH}_{2} \mathrm{Cl}_{2}, 0^{\circ} \mathrm{C}$

4. Nal, acetone
Category

Synthesis of Natural

Products and

Potential Drugs

\section{Key words}

(士)-leonuketal

8,9-seco-labdane terpenoid

titanium catalysis

Shapiro reaction

fragmentation

gold catalysis

spiroketalization<smiles>CC1(C)C2CCC1(C)C(CO[SH](=O)(F)F)C2(O)CCC1=C(O)COC1=O</smiles>

H
$\mathrm{TsOH} \cdot \mathrm{H}_{2} \mathrm{O}(10 \mathrm{~mol} \%)$ $\frac{\mathrm{MeOH}, \text { then } \mathrm{K}_{2} \mathrm{CO}_{3}}{84 \%}$<smiles>CCOC(=O)C(CC#CC1(O)C2CCC(C2)C1(C)C)C(=O)COC(C)C</smiles>

\section{$\mathrm{NaH}, \mathbf{F}, \mathrm{THF}$ $\frac{0{ }^{\circ} \mathbf{C} \text { to r.t., then } \mathbf{E}}{69 \% \text { from } \mathbf{D}}$ $\mathrm{dr}=1: 1$}

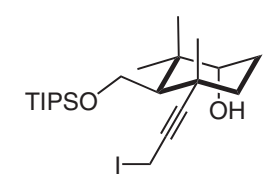

E

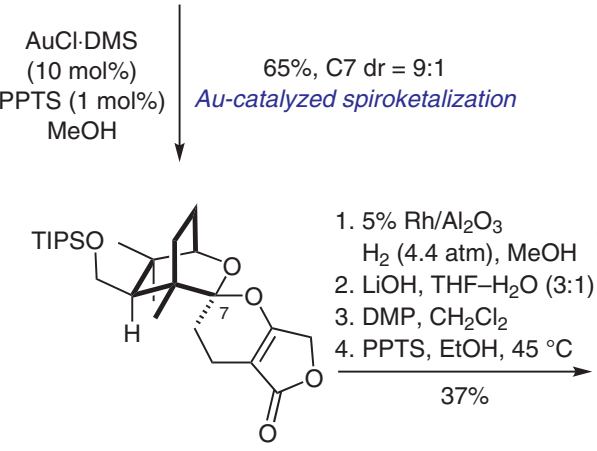

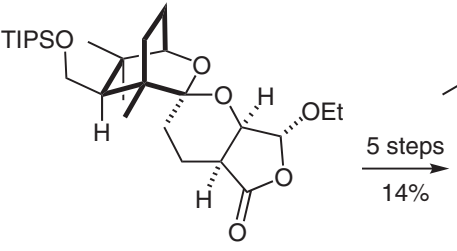

$J$

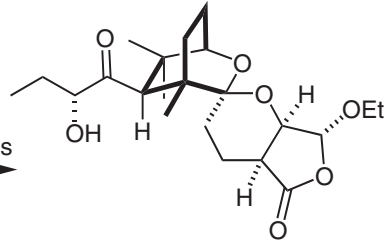

( \pm -Leonuketal
Significance: The tetracyclic 8,9-seco-labdane terpenoid (-)-leonuketal was isolated from the Chinese liverwort Leonurus japonicus and shows significant vasorelaxant activity against $\mathrm{KCl}$-induced contraction of rat aorta. The authors present the first total synthesis relying on a Ti-mediated cyclization, a Shapiro reaction-fragmentation and an Au-catalyzed spirocyclization.
Comment: Epoxide $\mathbf{A}$ was subjected to Ti-mediated radical cyclization to obtain bicyclic ketone $\mathbf{B}$. Shapiro reaction-fragmentation was applied to furnish alkyne $\mathbf{D}$. Alkylation of ketoester $\mathbf{F}$ with iodide $\mathbf{E}$ afforded alkyne $\mathbf{G}$ which was cyclized to butenolide H. Au-catalyzed spirocyclization delivered spiroketal I which was transformed into the natural product in additional 9 steps. 